\title{
Defining and Developing Soft Capabilities within Defence
}

\author{
Leon D. Young ${ }^{a}$ \\ ${ }^{\text {a }}$ The University of New South Wales, Northcott Dve, Campbell, ACT \\ Email: 1.young@adfa.edu.au
}

\begin{abstract}
Capability is broadly agreed to mean the capacity or ability to achieve an effect. In military circles, effect is more precisely defined as desired operational effect. This is to ensure that capability has a purpose. A certain quantifiable usefulness that allows capabilities to be deconstructed, costed, compared and developed. Capability development, certainly within the well-recognised capability life cycle model used in various militaries and business, is founded on the premise that a capability is measurable. They are hard, tangible and generally easily recognised. But what about the capabilities that are not obvious? How do we describe those capabilities that generate tangible effects however are not themselves directly quantifiable?
\end{abstract}

Quantifiable hard capabilities are simple enough to understand, though perhaps complicated to develop. When designing and developing hard capabilities, organisations are travelling down a well-travelled highway lined with information, models, exemplars and warnings. A model commonly used to describe the development of capability, to achieve a specific effect, describes the Fundamental Inputs to Capability (FIC). FICs are used to describe the building blocks required for specific capabilities and, while they vary slightly between models, generally include people, organisational design, facilities, support structures, other major systems, common or collective training and policies. The use of these labels is a convenient way to understand the important building blocks however are FICs common across all types of capabilities?

Soft capability though, whilst intuitively understood by many, is not well defined in current literature. This paper seeks to present a synthesised understanding of soft capability from a range of subject matter experts in capability development. The study explored the characteristics, inputs and examples of soft capability as understood by experts in the field. The results of the research demonstrate that soft capability is different to other capability types commonly referred to in literature. The conclusion is that soft capability is a valid capability type that has, to date, been ignored in literature.

Keywords: Soft capability, capability development, military capability, soft operations research, operational effects 


\section{INTRODUCTION}

Quantifiable hard capabilities are generally simple to understand, though perhaps complicated to develop. When designing and developing hard capabilities, organisations are travelling down a well-travelled highway lined with information, models, exemplars and warnings. Capability development, certainly within the wellrecognised capability life cycle model used in various militaries and business, is founded on the premise that a capability is measurable. They are hard, tangible and generally easily recognised. But what about the capabilities that are not obvious? How do we describe those capabilities that generate tangible effects however are not themselves directly quantifiable? This research forms part of a wider project into understanding the strategic thinking capability. As a capability, strategic thinking appeared to be ill-defined, difficult to measure and even more difficult to model. This seemed to reflect the lack of research into soft capabilities. The intent of this research was to explore soft capabilities in relation to related fields in current literature.

\section{CURRENT Understanding OF CAPABILITY}

In business terms, capability is viewed as a measure of the ability of an entity to achieve its objectives, especially in relation to its mission (BusinessDictionary.com, 2015). Objectives can be restated as the "desired operational effect" where an operational effect may be defined or described in terms of the nature of the effect and of how, when, where and for how long it is produced (Capability Development Group, 2014, p.2). This narrowing of the definition appears to ensure that specific capabilities have a purpose. A certain quantifiable usefulness that allows capabilities to be de-constructed, costed, compared and developed. Capability is simply the capacity to achieve an effect (Gaidow et al., 2006; Jagacinski and Nicholls, 1984, p.3).

The desired effect (or ends), within a military context, is to defeat an adversary. This is achieved, according to the Australian Armys philosophy, by reducing the enemys will to fight (Army, 2014, p.2). While the effect is cognitive in nature, it is achieved through predominantly physical means. It can be stated then that the physical actions required to reduce the enemy will are the means, not objectives, to generate soft effects.

Understandably then, given the large investment that goes into these capabilities, the focus of capability development is on physical means (such as Joint Strike Fighter or Air-Warfare Destroyers). As a result, while there is an acknowledgement of softer capabilities, the Defence Capability Development Handbook 2014, and the various capability development models, focus almost exclusively on physical capabilities (Capability Development Group, 2014; Helfat and Peteraf, 2003). This focus flows across into capability analysis, for example Thomson and Davies (2008) report on Australian Army capabilities only provided information of equipment. As a final consequence, there exists a set of capabilities that appear to be forgotten and are then not integrated into the wider available capability set. Existing concepts such as soft power, dynamic capabilities and knowledge management also appear to acknowledge soft capabilities.

Soft power - Within the geo-political sphere Nye (2004) coined the term "soft power" when he referred to the ability to change an entitys preferences and thus create a longer-lasting change in your favour. Soft power started to appear in literature from 1990 and was used to differentiate two types of power (Huang and Ding, 2006). Hard power generally referred to coercion and inducement while soft power referred to those intangible resources that invited others to want what you had. The argument is that hard power can be used to make an entity do something however soft power is able to change the entitys preferences and thus creating a longer lasting change in your favour. This exact point is mentioned in Brigadier Jans (2014) manuscript on Defence leadership when he states that "Senior leaders can certainly influence what people do but they are usually much less successful in shaping how people think and feel". Hence the importance in soft power lies in its capacity to influence preference. Soft Power, it seems, is difficult to evaluate as it is a less direct and visible source of a nations influence compared to hard power (Melissen and Lee, 2011, p.16). Soft power then could fit our description of soft capabilities however appears only available to nation states.

Dynamic capability - A study by Barreto (2010) provides a very good overview of Dynamic Capabilities. Using his work it is possible to attribute two characteristics to Dynamic Capabilities: (1) Capacity to develop, integrate, modify and reconfigure internal organisational processes, resource base or operational capability; and (2) the capacity to respond to the environment. According to Ambrosini and Bowman (2009), the use of dynamic and capability can create a misunderstanding of the term. If taken individually the words imply 
that the capability is dynamic when in fact the dynamism refers to the environment that dynamic capabilities respond to. Again, the description of dynamic capabilities appear to match soft capabilities but only in part, as they only seem to effect the organisation internal and do not seem to produce an external effect.

Knowledge management - The idea that knowledge should be treated as a significant organisational resource has grown in importance in recent times. Knowledge could be viewed as a capability as it has the potential to influence future action. The view espoused by Alavi and Leidner (2001), however is that knowledge, as a capability, is not the capacity for specific action rather the capacity to use information. As an internal capability, it can be developed through the integration of organisational directives, routines and specialised teams. Much of the literature on knowledge management, the highly relevant piece from Tanriverdi (2005) is a great exemplar, concentrates on the appropriate capture, internal distribution and integration of knowledge through an Information Technology lens. Like dynamic capability, knowledge management could be a soft capability but does not seem to encompass the full spectrum of effects one would expect.

The previous three concepts illustrate that there is an appreciation of softer capabilities. The descriptions do not appear to fully encompass the range of capabilities we would expect to label as 'soft' and we do not have a capability development model for soft capabilities per se. We do see soft capabilities can be developed through training, personnel, knowledge, etc. This is a horizontal integration exercise, while hard capabilities appear to be vertically integrated. Thus, while we acknowledge they are already considered, we conjecture that soft capabilities need a similar development model to that used for hard capabilities. Similarly soft power, dynamic capabilities and knowledge management have a strategic focus. We conjecture that by focusing on a soft capability development model, we can better understand and manage the logical integration of operational effects from soft capabilities to the grand strategic effects that ultimately creates soft power.

\section{Exploring Soft CAPABility - Methodology}

While there did not appear to be a common understanding or foundation of knowledge on soft capabilities, a contemporary understanding, based on experts within the field, would clarify any perceived differences to the other related fields of research. A qualitative research methodology was chosen as it allowed a conceptual framework to be elicited from a focused population who were familiar with the topic and could potentially talk from a position of strength (Flick, 2009). Semi-structured interviews allowed the capture of data rich in detail (Kvale and Brinkmann, 2009). The interviews were recorded and care was taken to ensure that the resulting transcription faithfully represented the intended meaning. The transcriptions were analysed using well-established qualitative research methods (including word maps, codification and influence diagrams). The result was a series of thematic nodes. Exploration of the themes would then develop a contemporary understanding of soft capability. The resultant characteristics were subsequently reviewed by participants to mitigate against researcher bias.

As the research depended heavily upon the data generated from the participants it was critical to ensure that participants understood enough to contribute and broadly represented the population. Participants were required to be subject matter experts (SME) in the field of capability development and sourced from within the Australian Defence Organisation as a recognised leader in the field. Due to the relatively small population size, selection bias was mitigated by seeking balanced participation from across service, organisation and position. Sample size was based on the Mason (2010) concept of saturation.

\section{Exploring Soft CAPABility - Results}

Figure 1 illustrates the number of nodes created from the transcripts and demonstrates that saturation was achieved after only six interviews. This finding is consistent with Mason (2010) that homogeneous populations can yield sufficient results after very low sample size. The capability development SME population is an example of such a population due to similar organisational practices, training and reward systems. Figure 2 illustrates the sample characteristics.

The 86 nodes yielded four broad themes (Soft Capability Characteristics, Soft Capability Inputs, Soft Capability Examples and Hard Capability Examples) and several nodes that appeared, on the surface at least, to be unrelated. Included in this later group were nodes such as funding, quotes and appreciation of soft capability. These outliers were examined in detail to ensure they were sufficiently distinct to warrant their own theme. 


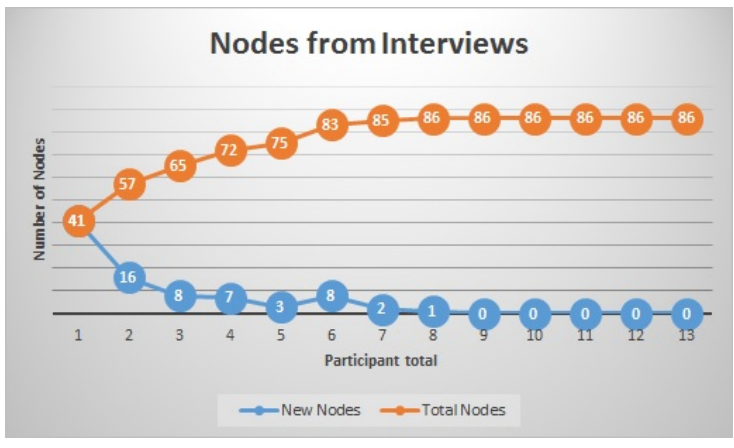

Figure 1. Number of Nodes created by participants

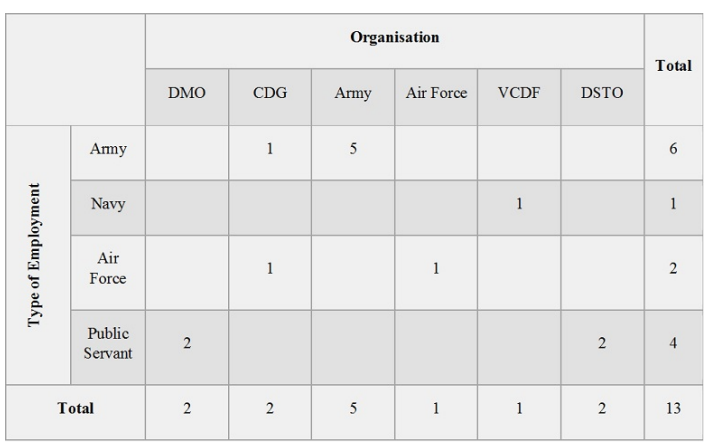

Figure 2. Sample Population

For instance the node "Economy" covered the same idea as "funding" and were combined and, on consideration, were moved under the "Soft Capability Characteristics" theme for further analysis. The result was five distinct themes (Appreciation, Characteristics, Inputs, Examples and Hard Capability). The first four will be discussed.

\subsection{Appreciation of Soft Capability}

"But we dont see them as capabilities. We don't see them as being important"

"I don't think Defence understands it at all"

It appears that there is an appreciation of soft capability within the Department of Defence even if the exact definition is unclear. The concern expressed by many of the participants was that, although they felt confident senior leaders appreciated the concept, they were not confident that this resulted in any tangible attempts to build or modify it. So while the organisation seems to understand the importance of "soft things" on operations and its resultant effect, there was also a universal acknowledgement that nothing was being done to develop the "soft things". The reason for this appeared to stem from the perceived intangibility of soft capabilities and thus the inherent difficulties in campaigning for a corresponding budget.

\subsection{Perceived Characteristics of Soft Capability}

The three most significant characteristics (evidenced in interviews and reviewed by participants) were the importance of people, the intangibility of the capability and lastly the ability to deliver an effect. The importance of people to the concept of soft capability should not be understated. Comments such as "people are the bearers of the capability" and "they are THE part of it" exemplify this majority position of the group. The predominance of people within soft capabilities also appeared to create a perceived level of complexity associated with the capability. This complexity is reflective of the Cynefin Framework developed by Kurtz and Snowden (2003). In this model one of the distinguishing elements between complicated and complex is the difficulties in modelling human behaviour. Others felt people were the recipient rather than the input into soft capability. In other words soft capability enhanced the potential of humans to contribute to an outcome or effect.

How soft capabilities actually delivered an effect was generally perceived to be very difficult. It is this intangibility that appeared to be one of the primary defining characteristics of soft capabilities. Comments such as "you can't grasp it" and "they're just not tangible" represent the common perception. This intangibility did not transmit across into the environment. Most of the participants felt that soft capabilities could generate an effect on an adversary however were undecided to what level. It was felt that this uncertainty reflected a lack of consideration by the participants on the cause-and-effect framework associated with soft capabilities.

\subsection{Provided Examples of Soft Capability}

While the participant population was relatively homogeneous in broad terms (i.e. they were all subject matter experts in capability development and had significant experience within the Defence Environment) the range of Soft Capability examples presented was quite interesting. This is understandable given the depth of their collected experiences within the Defence environment. By defining a Capability as the ability or capacity 
to do something and thus inferring that any capability, soft or hard, must be able to generate an effect, the provided examples were able to be filtered for consistency and feasibility. Two of the strongest examples of effects that could be achieved through soft capabilities are described below:

Shaping the Environment - Soft capabilities were perceived to be, in a sense, manipulative. They allow agents to not only understand the influences within an environment but also manipulate them, specifically other human agents. This could be seen in power exchanges on both the macro and micro levels. For instance it could refer to both the ability to shape a committee or organisational process to deliver a desired result and also to shaping a cultural environment to deliver a favourable national security outcome; and

Trust - This example provided a good contrast with hard capabilities. For instance a military force deployed into a threat environment will try to minimise the threat to their force. If the specific threat was indirect fire (mortar or missile) then the threat to the force could be minimised through early detection and warning systems (hard capability). Alternatively, or even concurrently, the force could look to build trust with the local community (soft capability) and thus reduce the likelihood of threat event. Both capabilities reduced the threat, though on different time scales. Development of trust could also be used internal to an organisation to develop that organisation's robustness.

\subsection{Suggested Inputs to soft Capabilities}

After discussing the characteristics of soft capabilities and exploring feasible examples the participants were asked to extrapolate the requisite inputs (or building blocks) for the capability. In many cases there was either a momentary look of panic or a considerable pause. This indicated that most of the participants had not consciously considered developing these soft capabilities and were having to evaluate the requirements on the spot. The final seven inputs broadly resembled the previously discussed FIC. The clear exception was the exclusion of the supply, support and major systems FIC. Human Resource Management was included as a separate input however this could be covered within the normal command and management FIC. Human Performance was also mentioned strongly but it could also easily be included within an expanded Personnel FIC.

\section{Discussion OF RESEARCH}

\subsection{Apparent Soft Capability Characteristics}

Two of the characteristics (people and intangible) dominated the discussions however this appeared to reflect the reality of the environment these capabilities are expected to operate in. Specifically a complex environment where the agents are not acting in a rational and pre-determined manner. People were seen to be the dominant and defining characteristic of soft capability. This is not really surprising because all capabilities appear to require an agent to produce the effect. In hard capabilities that often appears to be a tangible system or platform where people are only part of the system. As one of the participants phrased it, "people are the bearers" of soft capability. In classic capability development the view is to "man the equipment" however soft capability seems to reflect the older adage of "equipping the man". People then are the agents that generate the effect.

The interviews demonstrated that soft capability is perceived as different to hard capability. This perceived difference was often based on the idea that soft capability is intangible however this intangibility did not prevent soft capabilities from generating an effect. Any classic physicist would shudder at the previous statement, for how could an intangibility produce a tangible outcome? On exploration it appears that the intangibility nature of soft capability is really a reflection of the (1) complexity of the capability and (2) the inability to quantify the capability using existing tool sets. In other words the cause-and-effect is poorly understood. The complexity is merely a perception that reflects the difficulties in modelling the environment and the agents operating within that environment. The reliance upon people though is a constant and should be acknowledged within any soft capability modelling.

The interwoven theme of the complexity of the environment within which soft capabilities operated in was interesting. Certainly it followed the dominate thought that people were an essential requirement of soft capability and, as systems that include fluctuating societal norms could be classified as complex, this made 
sense in the Kurtz and Snowden (2003) Cynefin Model. The problem is that the complex environment is not really unique to soft capabilities, particularly in a Defence context. As one of the participants stated "The nature of war ... is enduring. It is basically a clash of wills." Thus any capability (hard or soft) used to pursue war-like goals would, in this context, be involved in a clash of wills. This means humans (at least until fully capable and wilful Artificial Intelligence is developed). Hence it would be fair to say that all capabilities generate an effect within a complex environment.

\subsection{Inputs to soft capability}

It is fairly clear the inputs are not significantly different between hard and soft capability to require a new FIC model. There was significant difference in emphasis on FIC. Specifically the central requirement for people in soft capabilities and major systems for hard capabilities. It is important to recall here that FIC are just convenient labels used to classify specific combinations of resources. The base resource type does not change, just the combinations. So while different FIC could be introduced into the model it does not seem to add value to the overall understanding of soft capability. Interestingly it appeared that soft capability could operate in isolation to hard capability however the reverse was not exactly true. For example a trust capability could directly affect an adversary however an air-warfare destroyer requires the full complement of FIC and were considerably enhanced through the influence of soft capabilities. This dilemma would require further research.

\subsection{Ability of Soft Capabilities to deliver an effect}

Hard capabilities are designed to deliver an effect. This research demonstrated that soft capabilities are perceived to also be able to generate an effect. While the effect could be the same or different, it is measurable. The measure may differ slightly however the examples provided showed that soft capability could generate comparable effects with hard capabilities. We have seen that both soft and hard capabilities work together. While a capability is defined by the ability or capacity to produce an effect, it is not useful unless that effect is desired. Hence it requires purpose.

\section{Comparing Soft Capabilities with Soft Power, Dynamic Capabilities and Knowl- EDge Management}

Dynamic capabilities initially appeared to strongly resemble soft capabilities due to its ability to influence hard capabilities and respond to the operational environment. However the significant difference was that soft capabilities could directly affect the external operational environment while dynamic capabilities were internally focused. They only looked to improve the organisational system or the hard capability. It would appear that the reason dynamic capabilities are similar is because they are actually a type of soft capability. A knowledge capability also appears to be a type of soft capability. It has a capacity to produce an effect (use information) that can improve an organisations performance however, like dynamic capabilities, it is internally focussed.

Soft power also appeared similar as it is often described as intangible and focussed on human preference. This inferred complex systems and people centric capabilities. On examination though it is clear that soft power is not talking about the capability per se rather it refers, in this context, to the effect generated. Often, on the international stage soft power utilises both hard and soft capabilities to manipulate other agents in the environment. Thus soft power is merely an example of the effect generated within the environment. The relationship between soft capability and soft power is comparable to the relationship between hard power and hard capabilities.

\section{Conclusion}

This research sought to understand the nature of Soft Capability and see if it matches current literature. This was achieved through a qualitative study of subject matter experts within the capability development field, specifically within a leading organisation. The interviews clearly showed an intuitive understanding of soft capability that is not reflected in the current literature. This study demonstrated that while there was a high appreciation of Soft Capability within the organisation there was little actual progress in the area. It found that the perceived complexity of the soft capability operating environment (due to the preponderance of people in the capability) and the apparent intangibility of soft capabilities has led to an almost universal disregard to its development. Examination of the building blocks showed that Soft Capabilities can be described using contemporary capability development models however there is a significant different in emphasis. Hard 
capabilities are generally platform-centric and soft capabilities are people-centric. Interestingly, it appeared that Soft capabilities were able to affect the environment independently while hard capabilities could not.

The provided examples illustrated that soft capabilities were able to produce tangible effects in the environment that could be comparable to hard capability, though the delay seemed to be longer. Initially soft capabilities appeared to be indistinguishable from knowledge management, dynamic capabilities and soft power. It became readily apparent that knowledge management and dynamic capabilities were in fact a type of internally focussed soft capability, that sought to to change the system not the environment. Soft power on the other hand was an example of the effect that could be generated by soft capabilities. The relationship between soft capability and soft power is comparable to the relationship between hard power and hard capabilities. Soft Capabilities can now be defined by their reliance upon people to produce an effect.

This research provides an exploratory glimpse into the nature of soft capabilities. Further research could examine the relative costs-benefits between comparable soft and hard capabilities as this would allow the development of robust soft capability development business cases. Similarly the development of measures to understand the relative importance of the inputs, specifically the human performance component of the personnel FIC, would be extremely beneficial to the development of appropriate design models.

Acknowledgement-I would like to acknowledge the invaluable support of the Australian Defence Force in this research and the guidance provided by Prof Hussein Abbass at UNSW Canberra.

\section{REFERENCES}

Alavi, M. and D. E. Leidner (2001). Review: Knowledge management and knowledge management systems: Conceptual foundations and research issues. MIS quarterly, 107-136.

Ambrosini, V. and C. Bowman (2009). What are dynamic capabilities and are they a useful construct in strategic management? International Journal of Management Reviews 11(1), 29-49.

Army, A. (2014). The Australian Army: An Aide-Memoire. Directorate of Plans - Army. http://www.army.gov.au//media/Army/Our

Barreto, I. (2010). Dynamic capabilities: A review of past research and an agenda for the future. Journal of Management 36(1), 256-280.

BusinessDictionary.com (2015). capability. http://www.businessdictionary.com/definition/capability.html. accessed on 19 February 2015.

Capability Development Group (2014). Defence Capability Development Handbook 2014. Commonwealth of Australia.

Flick, U. (2009). An introduction to qualitative research. Sage.

Gaidow, S., S. Boey, and R. Egudo (2006). A review of the capability options development and analysis system and the role of risk management. Technical report, DTIC Document.

Helfat, C. E. and M. A. Peteraf (2003). The dynamic resource-based view: Capability lifecycles. Strategic management journal 24(10), 997-1010.

Huang, Y. and S. Ding (2006). Dragons underbelly: An analysis of chinas soft power. East Asia 23(4), $22-44$.

Jagacinski, C. M. and J. G. Nicholls (1984). Conceptions of ability and related affects in task involvement and ego involvement. Journal of Educational Psychology 76(5), 909.

Jans, B. N. (2014). New values, old basics: How leadership shapes support for inclusion. Canberra, Australia: Centre for Defence and Strategic Studies, Australian Defence College.

Kurtz, C. F. and D. J. Snowden (2003). The new dynamics of strategy: Sense-making in a complex and complicated world. IBM systems journal 42(3), 462-483.

Kvale, S. and S. Brinkmann (2009). Interviews: Learning the craft of qualitative research interviewing. Sage.

Mason, M. (2010). Sample size and saturation in phd studies using qualitative interviews. Forum Qualitative Sozialforschung / Forum: Qualitative Social Research 11(3).

Melissen, J. and S. J. Lee (2011). Public diplomacy and soft power in East Asia. Palgrave Macmillan.

Nye, J. S. (2004). Soft power: The means to success in world politics. PublicAffairs.

Tanriverdi, H. (2005). Information technology relatedness, knowledge management capability, and performance of multibusiness firms. MIS quarterly, 311-334.

Thomson, M. and A. Davies (2008). Adf capability review: Australian army. Technical report, Australian Strategic Policy Institute. 